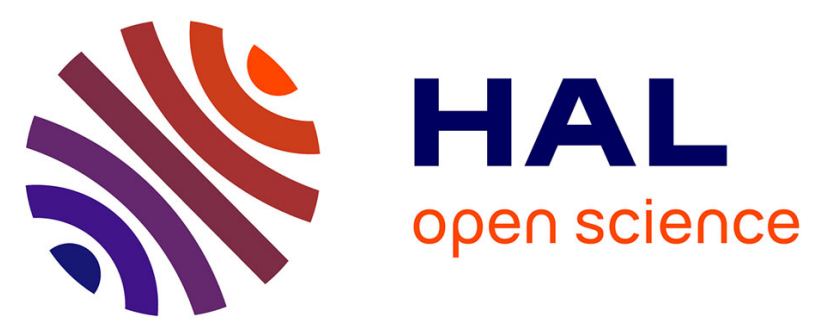

\title{
Effect of unequal levels of deformation and fragmentation on the electrochemical response of friction stir welded AA2024-T3 alloy
}

Fernanda Martins Queiroz, Uyime Donatus, Oscar Maurício Prada Ramirez,

João Victor de Sousa Araujo, Bárbara Victoria Gonçalves de Viveiros, Sviatlana Lamaka, Mikhail Zheludkevich, Mohammad Masoumi, Vincent Vivier, Isolda Costa, et al.

\section{- To cite this version:}

Fernanda Martins Queiroz, Uyime Donatus, Oscar Maurício Prada Ramirez, João Victor de Sousa Araujo, Bárbara Victoria Gonçalves de Viveiros, et al.. Effect of unequal levels of deformation and fragmentation on the electrochemical response of friction stir welded AA2024-T3 alloy. Electrochimica Acta, 2019, 313, pp.271-281. 10.1016/j.electacta.2019.04.137 . hal-02137721

\section{HAL Id: hal-02137721 \\ https: / hal.sorbonne-universite.fr/hal-02137721}

Submitted on 3 Jun 2019

HAL is a multi-disciplinary open access archive for the deposit and dissemination of scientific research documents, whether they are published or not. The documents may come from teaching and research institutions in France or abroad, or from public or private research centers.
L'archive ouverte pluridisciplinaire HAL, est destinée au dépôt et à la diffusion de documents scientifiques de niveau recherche, publiés ou non, émanant des établissements d'enseignement et de recherche français ou étrangers, des laboratoires publics ou privés. 


\title{
Effect of Unequal Levels of Deformation and Fragmentation on the
}

\section{Electrochemical Response of Friction Stir Welded AA2024-T3 alloy}

Fernanda Martins Queiroz $^{\mathrm{a}^{*}}$, Uyime Donatus ${ }^{\mathrm{b}}$, Oscar Maurício Prada Ramirez ${ }^{\mathrm{a}}$, João Victor de Sousa Araujo ${ }^{\mathrm{b}}$, Bárbara Victoria.Gonçalves de Viveiros ${ }^{\mathrm{b}}$, Sviatlana Lamaka ${ }^{\mathrm{c}}$, Mikhail Zheludkevich $^{\mathrm{c}}$, Mohammad Masoumi ${ }^{\mathrm{d}}$, Vincent Vivier ${ }^{\mathrm{e}}$, Isolda Costa ${ }^{\mathrm{b}}$, Hercílio Gomes de Melo ${ }^{\mathrm{a}}$

${ }^{a}$ Departamento de Engenharia Metalúrgica e de Materiais - Escola Politécnica da Universidade de São Paulo - EPUSP/PMT, Av. Professor Mello Moraes, 2463, São Paulo, SP, Brazil

${ }^{\mathrm{b}}$ Centro de Ciências e Tecnologia de Materiais - Instituto de Pesquisas Energéticas e Nucleares - IPEN/CCTM, Av. Professor Lineu Prestes, 2242, São Paulo, SP, Brazil

${ }^{c}$ Magnesium Innovation Centre, Institute of Materials Research, Helmholtz-Zentrum Geesthacht, MagIC - HZG, Max-Planck-Str. 1, 21502 Geesthacht, Germany

${ }^{\mathrm{d}}$ Centro de Engenharia, Modelagem e Ciências Sociais Aplicadas - Universidade Federal do ABC - CECS/UFABC, Avenida dos Estados, 5001, Santo André, SP, Brazil

${ }^{\mathrm{e}}$ Centre National de la Recherche Scientifique - CNRS - Sorbonne Université, F75005, Paris, France

*Corresponding author Email : mqfernanda@usp.br

\begin{abstract}
The effect of unequal levels of deformation and fragmentation on the electrochemical response of friction stir welded AA2024-T3 has been investigated using electron backscatter diffraction, scanning electron microscopy, global and localized electrochemical techniques. The friction stir welding process introduced unequal distribution and fragmentation of coarse intermetallic particles, such that more clusters
\end{abstract}


of these particles were present in the thermomechanically affected zone of the weld. This resulted in a higher susceptibility to severe localized corrosion in this region when compared with the others. Results from electrochemical measurements using a microcell could not be clearly correlated with the corrosion behaviour because no significant differences were observed using these techniques. On the other hand, LEIS and SVET results were in agreement and correlated with the corrosion evolution at the surfaces of the tested samples.

Keywords: Local electrochemical techniques; Friction stir welding; Localized corrosion; Clustering.

\section{Introduction}

In the last two decades, interest in the understanding of the initiation and propagation of severe localized corrosion (SLC) in $\mathrm{Al}$ alloys has risen exponentially due to an increasing demand for lightweight materials but also due to the advent of the friction stir welding (FSW) process. The corrosion behaviour of aluminium alloys, especially when welded, is quite complex [1-3] . Generally, the microstructural factors responsible for the initiation and propagation of SLC vary significantly for different alloys and temper conditions. An alteration in the temper condition of an alloy can significantly alter both the mode and rate of SLC. Thus, every case is unique, and generalization should not be made even if the alloys are from the same series.

A thermomechanical process, such as the FSW, alters the temper condition of the parent metal and produces distinct variations across the affected regions of the material. Consequently, macro and micro galvanic coupling activities are enhanced in the alloy. Also, it is interesting to note that modifications in the FSW parameters can alter the corrosion susceptibility of an alloy [4]. This is because the modifications introduce different degrees of deformation and thermal cycles. 
Additionally, the FSW process is asymmetrical and produces unequal degrees of deformation and peak temperatures on either side of the weld. The advancing side (AS) of the weld experiences higher temperatures compared with the retreating side (RS) $[5,6]$, and the microstructural modifications are always different in the two sides. Consequently, the thermomechanically affected zone (TMAZ) in the AS exhibits a different microstructure from the TMAZ in the RS, thus meaning that the width of the heat affected zone (HAZ) on either side will be different. To further confirm the asymmetry of friction stir welds, the stir zone (SZ) stems from the AS and shares a sharp boundary with the TMAZ at this side of the weld but with a diffuse boundary in the RS. All of these zones, alongside the base metal (BM), exhibit distinct microstructural features and consequently different mechanical and electrochemical responses.

For the AA2024 alloy investigated in this study, the formation and development of SLC have been attributed to clusters of coarse $\mathrm{Cu}$-rich particles [7-11] and grain stored energy [12]. Because the FSW process is asymmetrical, unequal distribution and clustering of the coarse $\mathrm{Cu}$-rich particles and different levels of grain-stored energies (consequent of the different degrees of deformation) are introduced across the weldment of the AA2024 alloy. Thus, a combination of these two factors is expected to control the corrosion susceptibility of the friction stir welded material. This combination has not yet been shown by the reports available in the literature. However, there are reports revealing the regions that are most susceptible to corrosion and the modes of corrosion in different weldments of the AA2024 alloy. Bousquet et al. [13] showed that the HAZ of the AA2024-T3 alloy was the most susceptible to intergranular corrosion in an ASTM G110 solution, because of the presence of continuous $\mathrm{S}^{\prime}(\mathrm{S})$ particles at the grain boundaries of the HAZ closest to the TMAZ. Conversely, Jariyaboon et al. [4] revealed 
that the most susceptible regions could either be the HAZ, SZ or both depending on the welding parameters in the same ASTM G110 test solution. The susceptibility observed was also associated with the sensitization of the grain boundaries in these regions. Kang et al. [14] observed pitting corrosion across the entire surface of the weld joint (using an EXCO solution) and argued that the degree of pitting was higher for the shoulder active region than the other regions. Nonetheless, it appears that there is no general agreement on the region that is the most susceptible to corrosion in the friction stir weldments of the AA2024 alloy; and no report has correlated the observed corrosion susceptibilities with clustering effect of the coarse $\mathrm{Cu}$-rich particles and grain stored energy. A possible reason for this may be the aggressiveness of the solutions employed. Less aggressive media may be required to allow an easy correlation of these factors with the corrosion susceptibility of the weldment of the AA2024 alloy. Furthermore, there is also no detailed report in the literature showing the differences between the local electrochemical responses of the AS and RS of the weld in a chloride-containing environment, and only the work of Squillace et al. [15], who employed potentiodynamic polarization and electrochemical impedance techniques, showed that the RS of the weld is nobler compared with the AS. Indeed, this finding is in contrast to what is observed in the present study as will be presented later. Also, experience has shown that the sole use of electrochemical techniques may not be sufficient to conclude on the corrosion susceptibility of friction stir welds of $\mathrm{Al}$ alloys, and for the base metal (BM) of $\mathrm{Al}$ alloys in general. Complementary information is needed for fully-fledged analysis of the factors controlling the corrosion of specific regions of friction stir weldments. The development of techniques for in situ characterization of localized electrochemical activities has helped to add more insight into the understanding of SLC initiation and propagation. A few studies have successfully employed techniques such as scanning 
electrochemical microscopy (SECM) [16], scanning vibrating electrode technique (SVET) [17-19] and localized electrochemical impedance spectroscopy (LEIS) [20] to reveal the corrosion modes and the regions that are most susceptible to corrosion in friction stir weldments. Thus, in this work, localized electrochemical techniques including the SVET and LEIS have been employed to reveal the difference between the corrosion behaviour of the advancing and retreating sides of the friction stir weldment of the AA2024-T3 alloy. The results obtained have been correlated with those of global electrochemical techniques and microstructural features, taking into account the asymmetric effects of the friction stir welding process.

\section{Experimental}

Friction stir welded plates, $2 \mathrm{~mm}$, of AA 2024-T3 alloy $(\mathrm{Mg} 1.6, \mathrm{Cu} 4.8, \mathrm{Fe} \mathrm{0.22,} \mathrm{Mn}$ 0.64, Si 0.19, Zn 0.08, P 0.03, S 0.02, Ca 0.04, Ti 0.05, Cr 0.04, Al Bal (wt \%)) were provided by a Brazilian company. The welding procedure is proprietary and employs a ratio between the tool speed rotation $(\mathrm{rpm})$ and the travel speed $(\mathrm{mm} / \mathrm{min})$ of 2.43 , which lies within the limits of 1.39 and 6.4 used by Jariyaboon et al. [4] in their investigation of the influence of the welding parameters on the corrosion behaviour of friction stir welded AA2024-T3 alloy. A schematic representation of the FSW process showing both the rotation and travel direction of the welding tool is presented in Figure 1.

Prior to corrosion tests and microstructural examinations, the surfaces of the welded samples were sequentially ground with $\mathrm{SiC}$ abrasive paper (grits size of 120, 500, 800, 1200 and 2500) and then polished with $1 \mu \mathrm{m}$ diamond suspension. For optical microscopy and electron backscatter diffraction (EBSD) analysis samples were finished with water free OPS silicon oxide polishing suspension. A Leica DMI 5000 light optical microscope was used to obtain the micrographs and show the morphologies of the 
grains across the weldment. The scanning electron microscopy (SEM) investigation, before and after immersion test, was performed using a Field Emission Scanning Electron Microscope (Inspect - F50) equipped with an energy dispersive X-ray spectroscopy (EDS) detector and a Zeiss FEG-SEM Ultra 55 microscope equipped with a Hikari detector and a TSL-OIM software package for electron backscatter diffraction (EBSD) analysis. The EBSD measurements were performed on an area of $100 \mu \mathrm{m} \times$ $100 \mu \mathrm{m}$, with a step size of $0.1 \mu \mathrm{m}$. OIM and MTEX softwares were used for the analyses of the data obtained.

Corrosion tests were performed using agar-visualization, local electrochemical impedance spectroscopy (LEIS) in the mapping mode (LEIM) and scanning vibrating electrode technique (SVET). A microcell with $0.035 \mathrm{~cm}^{2}$ of exposed area was used to perform open circuit potential (OCP) and electrochemical impedance spectroscopy (EIS) measurements. The microcell was built by drilling a cylindrical aperture in an acrylic plate, which was pressed against the flat surface of the sample using regulating screws, thus exposing only the selected weld zone to the test electrolyte. An O-ring was used to prevent leakage of the electrolyte.

Agar gel was prepared by adding $3 \mathrm{~g}$ of agar-agar powder and $7 \mathrm{~mL}$ of universal indicator into $100 \mathrm{~mL}$ of boiling $3.0 \% \mathrm{NaCl}$ solution. The solution was allowed to cool to a viscous state before being poured on the exposed sample surface. Macrographs were obtained at selected intervals with a digital camera to register the colour change as a function of time.

LEIS experiments in the mapping mode (LEIM) were carried out using a commercial Ametek ${ }^{\circledR}$ VS-LEIS system, controlled with the VerScan ${ }^{\circledR}$ Software. The scanned area was $1.05 \mathrm{~cm}^{2}$ and the maps were obtained at a single frequency of $5 \mathrm{~Hz}$ with a perturbation amplitude of $30 \mathrm{mV}(\mathrm{rms})$ and a step size of $200 \mu \mathrm{m}$. The experiments 
were performed in $\mathrm{Na}_{2} \mathrm{SO}_{4} 0.1 \mathrm{M}+\mathrm{NaCl} 1 \mathrm{mM}$ using an $\mathrm{Ag} / \mathrm{AgCl}$ electrode as the reference and a Pt ring as the counter electrode. Diagrams were regularly acquired up to $24 \mathrm{~h}$ of immersion. The frequency at which the maps were acquired was chosen according to the literature, and was set at a value that can provide information about the contribution of localized corrosion to the overall impedance [21].

OCP and EIS experiments were recorded using a microcell in the same solution as LEIS by means of a VersaStat $3 \mathrm{~F}$ potentiostat/galvanostat coupled to a frequency response analyser (FRA), controlled using the VersaStudio ${ }^{\circledR}$ software. OCP measurements were obtained up to 1 hour of immersion for the BM and all the weld zones and up to 24 hours for the TMAZ AS and RS. For these two latter regions, EIS diagrams were regularly recorded within the $24 \mathrm{~h}$ duration of the test. The diagrams were acquired in the frequency range from $10^{4}$ to $10^{-2} \mathrm{~Hz}$, using a perturbation amplitude of $10 \mathrm{mV}(\mathrm{rms})$ and at an acquisition rate of 10 points per decade. For both measurements, an $\mathrm{Ag} / \mathrm{AgCl}$ microelectrode was used as a reference and a platinum wire as counter electrode.

SVET experiments were performed in $\mathrm{NaCl} 0.005 \mathrm{M}$ using an Applicable Electronic device controlled by the ASET 4.0 software. For the measurements, the probe was positioned at $100 \pm 5 \mu \mathrm{m}$ from the sample surface, and maps were acquired using a vibration amplitude of $19 \mu \mathrm{m}$, at frequencies of $174 \mathrm{~Hz}$ and $73 \mathrm{~Hz}$ in the $\mathrm{X}$ and $\mathrm{Y}$ axis directions, respectively. Maps were recorded every $2 \mathrm{~h}$ up to $24 \mathrm{~h}$, using a step of 200 $\mu \mathrm{m}$, totalizing $71 \times 26$ points per map. The distribution of local current density was recorded in $10 \mathrm{~mL}$ of test electrolyte which was constantly pumped, at a flow rate of 0.2 $\mathrm{L} / \mathrm{h}$, from a reservoir with fresh solution, thus maintaining constant the concentration and the conductivity during the test. Further experimental details on the these measurements can be found in the work of de Sousa Araujo et al. [22].

\section{Results and Discussion}




\subsection{Microstructure}

Figure 2 shows the cross-section of the whole welded joint where the SZ and the TMAZ at the AS and RS are identified. In order to explore the variation of crystallographic orientations and mesotexture changes due to the FSW process and their possible correlation with corrosion activity, EBSD measurements were performed in the areas identified by red boxes in the Figure, and their results are discussed in detail in the following paragraphs. The EBSD of the base material was recorded $10 \mathrm{~mm}$ away from the SZ towards the RS.

Grain boundaries, orientation images, and Kernel average misorientation maps of each of the regions highlighted in Figure 2 are presented in Figure 3. The average size of $\alpha$ Al grains in the parent microstructure of the base AA2024 (Figure 3(a)) was estimated to be about $7.5 \mu \mathrm{m}$. The microstructure is characterized by coarse grains with a large number of high angle boundaries. Also, some precipitates such as $\mathrm{CuAl}_{2}$ and $\mathrm{CuAl}_{2} \mathrm{Mg}$ were found in the grains and at the grains boundaries. Ultrafine equiaxed grains (smaller than $1 \mu \mathrm{m}$ ) were observed in the stir zone (Figure 3(b)). The severe plastic deformation during FSW with the associated high temperature enhanced dynamic recrystallization and grain refinement in this zone $[23,24]$. Low level of Kernel average misorientation values in the corresponding map also indicates the low level of accumulated dislocations due to the occurrence of dynamic recrystallization.

It is well-known that the solid-state FSW process induces severe plastic deformation at high temperatures (slightly below the Al melting point). Kandasamy et al. [25] reported that temperatures could reach $560{ }^{\circ} \mathrm{C}$ in the $\mathrm{SZ}$ of an AA7075 aluminum alloy during FSW. As mentioned above, this temperature is sufficiently high to promote full dynamic recrystallization in this zone, as shown in Figure 3(b). However, the temperature gradually decreases with joint distance. Teh et al. [26] reported that with 
complex geometry rotating tools, the amount of heat generated by surface friction is lower in the RS than in the AS. This is because the degree of deformation is always higher in the AS region when compared with the RS region since the tool interacts more with the BM at the AS. At the RS, the tool spins the already plasticized material around the pin axis to the trailing edge, forming a more heterogeneous region, as evidenced in the right side of Figure 2. Therefore, it is likely that additional heat flow enhances the dynamic recrystallization in the AS when compared with the RS.

To investigate the mesotexture changes in distinct regions, Figure 4 shows the variation of boundary types and Kernel average misorientation (KAM). Grain boundaries are generally divided into high angle boundaries (HABs) at which point-to-point misorientation is greater than $15^{\circ}$ and moderate angle boundaries (MABs) or subgrains $\left(5^{\circ}<\theta<15^{\circ}\right)$. Deformed grains are characterized by misorientation of less than $5^{\circ}$. As mentioned earlier, the intense plastic deformation at a higher temperature in the SZ formed a region with recrystallized ultrafine grain. Accordingly, the high fraction of HABs with a low number of MABs and deformed grains in the SZ indicate the development of non-deformed recrystallized grains with low stored energy. The same can be said of the TMAZ for the AS, howbeit, to a lower degree compared with the SZ. In contrast, a high number of deformed grains were identified in the TMAZ of the RS. This microstructure corresponds to a heavily distorted structure resulting from the material plastic flow during welding in the absence of dynamic recrystallization and recovery. In other words, plastic deformation in this region produced some crystallographic defects at the grain interior and at the grain boundaries which increased the stored energy and might influence its corrosion susceptibility. Figure 4 also shows that the variation of coincidence site lattice (CLS, or special boundaries) was negligible in all the zones. 
Figure 5 shows SEM images of the distribution and degree of fragmentation (as evidenced by the particle sizes) in the different zones of the weld, as a reference a micrograph of the BM far from the weld zone was also added (Figure 5(a)). Because the deformation is higher in the TMAZ(AS) (Figures 5(d) and (f)) and SZ (Figure 5b)), the coarse intermetallics (IMCs) in these zones are more fragmented and more evenly distributed in these regions compared with the TMAZ(RS) (Figures 5(e) and (g)). In the RS side of the weld, the lower degree of deformation and the flow of the plasticized material results in less fragmentation and increased clustering of the coarse IMCs in the direction of tool motion (Figures 5 (e) and (g)). Comparing the TMAZ(AS) and the TMAZ(RS) in Figures 5 (f) and 5 (g), it can be observed that in addition to the clustering and lower degree of fragmentation of the coarse IMC particles, there were, to a larger extent, particle-free regions in the vicinities of the coarse particles in the RS compared with the AS. These unequal degrees of fragmentation and clustering influence the extent to which these regions are susceptible to corrosion as will be discussed in the following. Concerning the HAZ (Figure 5(c)), as expected, IMC distribution is very similar to that exhibited by the BM (Figure 5(a)), confirming that this feature is not affected by the FSW process.

Generally, the AA2024 alloy is composed of Al-Cu-Mg, Al-Cu, Al-Cu-Fe, Al-Cu-Fe$\mathrm{Mn}-\mathrm{Si}$, and Al-Cu-Fe-Mn coarse IMC particles [10,27]. These particles present different electrochemical behaviours with respect to the matrix. The $\mathrm{Al}-\mathrm{Cu}-\mathrm{Mg}$, the $\mathrm{S}\left(\mathrm{Al}_{2} \mathrm{CuMg}\right)$ phase, is usually associated with the initiation of SLC in this alloy $[7,28,29]$. However, other reports have revealed that clusters of $\mathrm{Cu}$-rich particles, other than the $\mathrm{S}$-phase, control the formation of stable pits in the AA2024 alloy [10]. As earlier detailed, friction stir welding results in fragmentation of these particles. The temperature reached during the process is usually high enough to cause the dissolution of the particles, 
except for the Fe and Mn-containing phases which are usually very stable [30]. Nonetheless, $\mathrm{Cu}$-rich precipitates are still present in the zones of the weld, as shown in Figure 5, and these are predominantly the anodic S-phase since this phase makes up for more than $60 \%$ of the coarse particles in the AA2024 alloy [29]. Thus, the heterogeneous clustering of these particles can significantly influence the corrosion behaviour of the zones.

\subsection{Corrosion}

Figure 6 shows the agar visualization test result. In this test, regions of low $\mathrm{pH}$ are associated with anodic activities due to the $\mathrm{H}^{+}$produced from hydrolysis of $\mathrm{Al}^{3+}$ [31]. In contrast, high $\mathrm{pH}$ regions are associated with cathodic activities involving the reduction of dissolved oxygen which produces hydroxyl ions. It must, however, be stated that galvanic interactions should be slightly lower because of ions entrapment by the gel and significantly slower diffusion of the released ions and oxygen. Nonetheless, the agar visualization technique, introduced by Isaacs et al. [31], gives an overview of the most susceptible or anodic region in the weldment. From these images, it can be seen that the TMAZ region of the RS was the most anodic zone in the whole weldment. Over the whole duration of the test ( 24 hours), no other region exhibited pronounced anodic activities. This was also confirmed by examining the surface after the removal of the agar gel (bottom macrograph).

To evaluate possible galvanic interactions across the weld zone, OCP measurements were obtained from the different regions of the weldment using a microcell. Results from the SZ and the regions of the AS were compared with those of the regions from the RS in a bid to establish the asymmetric effect of the FSW process on the galvanic events across the surface of the weldment. However, even though, just after immersion, the OCP of the TMAZ at the RS was clearly higher than the others, after $1 \mathrm{~h}$ immersion, 
the OCP results tend to show values within the same range, maximum difference of about $30 \mathrm{mV}$, for all the tested regions (as presented in Figure 7). Because the TMAZ(RS) was the most anodic region observed from the agar visualization results, the OCP of this zone was selectively compared with that of the TMAZ(AS) for 5 min after 24 hours immersion - Figure 8. From this result, the TMAZ(RS) with an average OCP value in the range of $-613 \mathrm{mV}$ was slightly anodic $(-26 \mathrm{mV})$ to the TMAZ(AS) with an average OCP value of $-587 \mathrm{mV}$. Taking into account the results presented in Figure 7, this indicates a similar evolution of the OCP at both TMAZ. Similarly, no significant differences were observed between the OCP of the TMAZ(RS) and the other zones. Therefore, the OCP differences do not appear to be large enough to drive strong galvanic interaction between adjacent regions of the weld, as evidenced in the agar-agar test. Based on this, electrochemical impedance spectroscopy (EIS) tests using the microcell were also conducted for these two zones (TMAZ(AS) and (RS)). Again, no significant differences were found as typified by the EIS spectra presented in Figure 9. Thus, the EIS behaviour was the same for these two zones for the frequency range employed.

As discussed above, establishing the galvanic effects by comparing the responses of the different weld regions from traditional electrochemical measurements using the microcell at each region was not evident in the conditions employed in this work. The tests were repeated more than 5 times in each case, and no inference regarding differences in the electrochemical responses of the weld zones could be drawn. This necessitated further investigation. Therefore, two local electrochemical techniques - the SVET and LEIS (in the mapping mode-LEIM) - were employed to understand the corrosion susceptibility of the weldment further. 
Figures 10-11 show the SVET results for the entire surface of the weldment. In the SVET maps, blue colours represent cathodic sites, whereas red/orange regions characterize the zones with stronger anodic activity. During the early hours of the SVET test, pronounced anodic activities were observed on both TMAZ regions; but the activities were higher in the TMAZ(RS), and the number of SLC sites were also higher in this region compared with the TMAZ(AS). Except for a few more sites which developed during the test, the trend was the same throughout the test. SEM images of the different regions of the surface after the test (Figure 11) confirm the formation of SLC in the regions that showed high anodic activities on the SVET map. A typical feature of SLC in aluminium alloys is the presence of corrosion rings around the SLC sites, and this is evident in Figures 11(b) and 11(d) which correspond to the regions of high anodic activities on the SVET maps. The current density values are high in these regions because of the prevalent ionic currents resulting from the stable pitting activities. The stable pitting activities, in this case, are associated with galvanic interactions between coupled intermetallic particles and the matrix, and this result in deeply penetrating intergranular attack. On the contrary, regions without corrosion rings (Figure 11(c)) presented low current density values because stable pitting did not occur in such regions. The prevalent features in such regions were the formation of cavities and trenches around coarse intermetallic particles, and these did not involve high ionic currents to be detected by SVET. These features are associated with the dark spots in the SEM images. As clearly shown, the regions with corrosion rings were present on the TMAZ of both sides. Thus, certain areas of the TMAZ(AS) were also susceptible to SLC. However, the most anodic region in the weldment is clearly the TMAZ(RS).

The LEIM diagrams presented in Figure 12 also revealed that the TMAZ(RS) region was the most susceptible region to SLC. Higher admittance modulus was recorded on 
the TMAZ(RS) region compared with the rest of the weld for the test period presented, since higher admittance modulus corresponds to lower impedance and ultimately lower resistance to corrosion. The LEIM results are in good agreement with the SVET and agar visualization results confirming that the TMAZ(RS) is the most susceptible zone to SLC. The agreement between the SVET and LEIM results shows that the LEIS is a very useful technique in the characterization of the electrochemical responses across the weldment of an alloy. Interestingly, the complementarity of these techniques can be explained as follows. The frequency used for the LEIS mapping allows differentiating more clearly the kinetics of the electrochemical processes taking place at each region of the sample surface, as it may vary with the microstructure, which, as previously demonstrated, is strongly affected by the FSW process, however it does not allow differentiating cathodic and anodic domains, whereas the SVET allowed to measure the dc-contribution of the current (contribution which is hardly measurable with LEIS), allowing to clearly identify anodic and cathodic sites.

To correlate these results with the microstructural modifications introduced by the FSW process, the surface of the welded sample was examined after the immersion tests. Figure 13 shows SEM images for the AS and RS of the welded sample after immersion in $0.1 \mathrm{M} \mathrm{NaCl}$ for 12 hours. The TMAZ(RS) was far more susceptible to SLC. The banded region of about $600 \mu \mathrm{m}$ in length was the most active domain for the formation of this type of corrosion. This region shares a boundary with the HAZ. Even for a shorter immersion time, ( $2 \mathrm{~h}$ - Figure 14$)$, it was already very clear that the attack was preferentially originated from this boundary, around $\mathrm{Cu}$-rich IMC particles (Figure 14(d)), in fully agreement with the SVET and LEIS maps.

As previously shown, the TMAZ(RS) presented the highest amount of low angle grain boundaries (subgrains). This resulted from the fact that this region was highly 
deformed, but the level of deformation was neither high enough nor was the peak temperature reached in this region sufficient to induce recrystallization. Thus, compared with the SZ and TMAZ(AS) where the degree of deformation and the temperature reached were sufficient to induce recrystallization to form stress-free grains, the TMAZ(RS) should exhibit a higher amount of stored energy. However, the typical hardness profiles of the friction stir weldment of this alloy $[4,13,29]$ contradict this theory. The hardness values of the TMAZ/HAZ boundaries (which are the regions for the preferential attack) are usually lower than those of the adjacent SZ, HAZ and BM. This means that the stored energy in this region cannot be associated with the observed preferential corrosion susceptibility. Moreover, Guerin et al. [32] carried out an extensive study to show the correlation of the level of grain boundary misorientation with corrosion susceptibility using the AA2050 alloy. It was observed that even though the proportion of subgrain boundaries was far higher than the proportion of high angle grain boundaries, only $30 \%$ of the corroded interfaces were subgrain boundaries while $70 \%$ were high angle grain boundaries. This shows that low angle grain boundaries (subgrains) are more resistant to corrosion. This result agrees with other works in the literature, such as the works of Bennett and Pickering [33] on ferritic and austenitic stainless steels and that of Kim et al. [34] on high purity aluminium. In essence, the preferential attack observed in the TMAZ(RS) of the AA2024-T3 weldment is not associated with grain stored energy, nor is it associated with the level of misorientation of the grain boundaries. Thus, a different factor has to be considered. In doing this, the corroded surfaces of the weldment were carefully examined after immersion tests. SEM images obtained from the HAZ-TMAZ-SZ(AS) and HAZ-TMAZ-SZ(RS) of the weldment after a 12 hours immersion period are presented in Figure 13. As with the case of the other corrosion results, a high number of SLC sites were preferentially 
situated in the TMAZ(RS), in a region adjacent to the HAZ as indicated in Figure 13(b). To monitor the exact preferred SLC sites in the TMAZ(RS), an immersion test was also performed for only two hours with SEM images taken afterwards (Figure 14). This time around, the SLC sites were at the early stage of development, and better observations can be made. Firstly, the attacks were, again, situated at the TMAZ(RS) region immediately adjacent the HAZ. A closer look suggests that the attacks were initiated at regions with clustered coarse IMC particles, as demonstrated by the EDS spectrum (Figure 14(d)). Figure 15 shows SEM images taken from selected SLC sites in the TMAZ(RS) region of the sample exposed for 12 hours. From these images, it is evident that the SLC attacks were initiated at regions with clusters of coarse IMC particles. In agreement with our previous result, more clusters of the coarse $\mathrm{Cu}$-rich IMC are found in the TMAZ(RS) compared with all the other regions of the weldment. Furthermore, because the level of fragmentation is lower (compared with the SZ and TMAZ(AS)), the TMAZ(RS) is composed of more coarse-particle free regions and regions with coarse particles. Clusters of $\mathrm{Cu}$-containing particles (particularly those of the $\mathrm{S}\left(\mathrm{Al}_{2} \mathrm{CuMg}\right)$ phase) have always been associated with the development of SLC in the AA2024 alloy. Zhou et al. [7] have shown that stable localized corrosion initiated at sites where large clusters of the S-phase were immediately beneath the alloy surface, and the attack propagated through the grain boundaries. Hughes et al. [8] also found a high number of particles within the corrosion rings associated with SLC suggesting that local clustering played a key role in the initiation and development of SLC. Knight et al. [35] and King et al. [11] reported similar findings and the corrosion pathways were also intergranular after the initiation at regions with clusters of coarse IMCs. The reports from these authors support the findings of this work revealing that clustering of coarse $\mathrm{Cu}$-rich particles is responsible for the initiation of SLC in the TMAZ(RS) of the AA2024-T3 
weldment. As evident, the attack also propagated through the grain boundaries after the initiation of attack at the clustered sites. Another interesting finding was the redeposition of $\mathrm{Cu}$ at the grain boundaries in the corroded region (Figures 15(c) and (d)). This re-deposition possibly enhanced the growth of the SLC through the interactions of the cathodic re-deposited $\mathrm{Cu}$ and the adjacent matrix. Furthermore, it is also possible that galvanic interactions occurred between the particle clusters and the particle-free regions to promote the formation of SLC.

\section{Conclusions}

1. Friction stir welding caused unequal distribution and clustering of the coarse intermetallic particles across the zones of friction stir welded AA2024-T3 alloy.

2. Grain stored energy and grain boundary misorientation did not affect the corrosion susceptibility.

3. Clusters of coarse intermetallic particles were more abundant in the TMAZ of the retreating side, and this resulted in the formation of more SLC sites in this region.

4. Electrochemical tests carried out with the microcell, such as OCP and EIS, were not sensitive enough for the explanation of the observed differences in the corrosion susceptibilities of the zones of the weldment.

5. LEIS and SVET results were in agreement and correlated well with the microstructural features and corrosion evolution at the surfaces of the tested samples of the weldment.

\section{Acknowledgements}

The authors are grateful to FAPESP for financial support (Proc. 2013/13235-6) to this research and for the grants of F.M.Queiroz (Proc. 2016/20572-7) and U. Donatus (2017/03095-3). O.M. Prada Ramirez and J. V. de Sousa Araujo are thankful, 
respectively, to CNPq grant 168625/2017-2 and CNPq 169569/2017-9. Engineer Petra Fisher from MagIC, Geesthacht is acknowledged for sample preparation and EBSD measurements as well as José Veríssimo S. dos Santos from LabMicro EPUSP/PMT, for SEM images.

\section{References}

[1] T. Dursun, C. Soutis, Recent developments in advanced aircraft aluminium alloys, Mater. Des. 56 (2014) 862-871. doi:10.1016/j.matdes.2013.12.002.

[2] D. Kim, Y. Choi, Y. Kim, S. Jung, Characteristics of Nanophase WC and WC-3 $\mathrm{wt} \%$ (Ni, Co, and Fe) Alloys Using a Rapid Sintering Process for the Application of Friction Stir Processing Tools, Adv. Mater. Sci. Eng. 2015 (2015) 1-9. doi:10.1155/2015/343619.

[3] S. Lomolino, R. Tovo, J. Dos Santos, On the fatigue behaviour and design curves of friction stir butt-welded Al alloys, Int. J. Fatigue. 27 (2005) 305-316. doi:10.1016/j.ijfatigue.2004.06.013.

[4] M. Jariyaboon, A.J. Davenport, R. Ambat, B.J. Connolly, S.W. Williams, D.A. Price, The effect of welding parameters on the corrosion behaviour of friction stir welded AA2024-T351, Corros. Sci. 49 (2007) 877-909. doi:10.1016/j.corsci.2006.05.038.

[5] R.S. Mishra, Z.Y. Ma, Friction stir welding and processing, Mater. Sci. Eng. R Reports. 50 (2005) 1-78. doi:10.1016/j.mser.2005.07.001.

[6] X. He, F. Gu, A. Ball, A review of numerical analysis of friction stir welding, Prog. Mater. Sci. 65 (2014) 1-66. doi:10.1016/j.pmatsci.2014.03.003.

[7] X. Zhou, C. Luo, T. Hashimoto, A.E. Hughes, G.E. Thompson, Study of localized corrosion in AA2024 aluminium alloy using electron tomography, Corros. Sci. 58 (2012) 299-306. doi:10.1016/j.corsci.2012.02.001. 
[8] A. Hughes, A. Boag, A. Glenn, Corrosion of AA2024-T3 Part II: Co-operative corrosion, Corros. .... 53 (2011) 27-39. doi:10.1016/j.corsci.2010.09.030.

[9] A. Boag, A. Hughes, A. Glenn, Corrosion of AA2024-T3 Part I: Localised corrosion of isolated IM particles, Corros. ... 53 (2011) 17-26. doi:10.1016/j.corsci.2010.09.009.

[10] A.E. Hughes, R. Parvizi, M. Forsyth, Microstructure and corrosion of AA2024, Corros. Rev. 33 (2015) 1-30. doi:10.1515/corrrev-2014-0039.

[11] P. King, I. Cole, P. Corrigan, A. Hughes, T. Muster, FIB/SEM study of AA2024 corrosion under a seawater drop: Part I, Corros. Sci. 53 (2011) 1086-1096. doi:10.1016/j.corsci.2010.12.004.

[12] C. Luo, X. Zhou, G.E. Thompson, A.E. Hughes, Observations of intergranular corrosion in AA2024-T351: The influence of grain stored energy, Corros. Sci. 61 (2012) 35-44. doi:10.1016/j.corsci.2012.04.005.

[13] E. Bousquet, A. Poulon-Quintin, M. Puiggali, O. Devos, M. Touzet, Relationship between microstructure, microhardness and corrosion sensitivity of an AA 2024T3 friction stir welded joint, Corros. Sci. 53 (2011) 3026-3034. doi:10.1016/j.corsci.2011.05.049.

[14] J. Kang, R. dong Fu, G. hong Luan, C. lin Dong, M. He, In-situ investigation on the pitting corrosion behavior of friction stir welded joint of AA2024-T3 aluminium alloy, Corros. Sci. $52 \quad$ (2010) 620-626. doi:10.1016/j.corsci.2009.10.027.

[15] A. Squillace, A. De Fenzo, G. Giorleo, F. Bellucci, A comparison between FSW and TIG welding techniques: Modifications of microstructure and pitting corrosion resistance in AA 2024-T3 butt joints, J. Mater. Process. Technol. 152 (2004) 97-105. doi:10.1016/j.jmatprotec.2004.03.022. 
[16] D. Sidane, E. Bousquet, O. Devos, M. Puiggali, M. Touzet, V. Vivier, Local electrochemical study of friction stir welded aluminum alloy assembly, J. Electroanal. Chem. 737 (2014) 206-211. doi:10.1016/j.jelechem.2014.06.025.

[17] J.R. Kish, N. Birbilis, E.M. McNally, C.F. Glover, X. Zhang, J.R. McDermid, G. Williams, Corrosion Performance of Friction Stir Linear Lap Welded AM60B Joints, JOM. 69 (2017) 2335-2344. doi:10.1007/s11837-017-2504-6.

[18] J.C.B. Bertoncello, S.M. Manhabosco, L.F.P. Dick, Corrosion study of the friction stir lap joint of AA7050-T76511 on AA2024-T3 using the scanning vibrating electrode technique, Corros. Sci. $94 \quad$ (2015) 359-367. doi:10.1016/j.corsci.2015.02.029.

[19] U. Donatus, B.V.G. de Viveiros, M.C. de Alencar, R.O. Ferreira, M.X. Milagre, I. Costa, B.V.G. de Viveiros, M.C. de Alencar, R.O. Ferreira, M.X. Milagre, I. Costa, Correlation between corrosion resistance, anodic hydrogen evolution and microhardness in friction stir weldment of AA2198 alloy, Mater. Charact. 144 (2018) 99-112. doi:10.1016/j.matchar.2018.07.004.

[20] C.P. de Abreu, I. Costa, H.G. de Melo, N. Pébère, B. Tribollet, V. Vivier, Multiscale Electrochemical Study of Welded Al Alloys Joined by Friction Stir Welding, J. Electrochem. Soc. $164 \quad$ (2017) C735-C746. doi:10.1149/2.0391713jes.

[21] J.B. Jorcin, E. Aragon, C. Merlatti, N. Pébère, Delaminated areas beneath organic coating: A local electrochemical impedance approach, Corros. Sci. 48 (2006) 1779-1790. doi:10.1016/j.corsci.2005.05.031.

[22] J.V. de Sousa Araujo, U. Donatus, F.M. Queiroz, M. Terada, M.X. Milagre, M.C. de Alencar, I. Costa, On the severe localized corrosion susceptibility of the AA2198-T851 alloy, Corros. Sci. $133 \quad$ (2018) 132-140. 
doi:10.1016/j.corsci.2018.01.028.

[23] S. Chen, X. Li, X. Jiang, T. Yuan, Y. Hu, The effect of microstructure on the mechanical properties of friction stir welded 5A06 Al Alloy, Mater. Sci. Eng. A. 735 (2018) 382-393. doi:10.1016/j.msea.2018.08.020.

[24] M.P. Miles, T.W. Nelson, C. Gunter, F.C. Liu, L. Fourment, T. Mathis, Predicting recrystallized grain size in friction stir processed 304L stainless steel, J. Mater. Sci. Technol. 8 (2018) 1254-1276. doi:10.1016/j.jmst.2018.10.021.

[25] J. Kandasamy, M.M. Hussain, S. Rajesham, An Investigation on the Effect of Rolling Direction on the IMC Formed During Friction Stir Welding of AA7075 Alloys, J. Mech. Eng. IV (2011) 44-54.

[26] N.J. Teh, H. Goddin, A. Whitaker, Developments in micro applications of friction stir welding, J. Weld. Join. 14 (2015) 221-238.

[27] A. Boag, R. Taylor, T. Muster, Stable pit formation on AA2024-T3 in a NaCl environment, Corros. .... 52 (2010) 90-103. doi:10.1016/j.corsci.2009.08.043.

[28] P. Leblanc, G.S. Frankel, A Study of Corrosion and Pitting Initiation of AA2024T3 Using Atomic Force Microscopy, J. Electrochem. Soc. 149 (2002) B239. doi:10.1149/1.1471546.

[29] N. Dimitrov, J. a. Mann, M. Vukmirovic, K. Sieradzki, Dealloying of Al[sub 2]CuMg in Alkaline Media, J. Electrochem. Soc. 147 (2000) 3283. doi:10.1149/1.1393896.

[30] A.K. Shukla, W.A. Baeslack, Effect of Process Conditions on Microstructure Evolution and Mechanical Properties of Friction Stir Welded Thin Sheet 2024T3, 6th Int. Symp. Frict. Stir Weld., 2006. (n.d.).

[31] H.S. Isaacs, G. Adzic, C.S. Jeffcoate, 2000 W.R. Whitney Award Lecture: Visualizing Corrosion, Corrosion. 56 (2000) 971-978. doi:10.5006/1.3294386. 
[32] M. Guérin, J. Alexis, E. Andrieu, L. Laffont, W. Lefebvre, G. Odemer, C. Blanc, Identification of the metallurgical parameters explaining the corrosion susceptibility in a 2050 aluminium alloy, Corros. Sci. 102 (2016) 291-300. doi:10.1016/j.corsci.2015.10.020.

[33] B.W. Bennett, H.W. Pickering, Effect of grain boundary structure on sensitization and corrosion of stainless steel, Metall. Trans. A. 22 (1991) 11171124. doi:10.1007/BF03325722.

[34] S.. Kim, U. Erb, K.. Aust, G. Palumbo, Grain boundary character distribution and intergranular corrosion behavior in high purity aluminum, Scr. Mater. 44 (2001) 835-839. doi:10.1016/S1359-6462(00)00682-5.

[35] S.P. Knight, M. Salagaras, A.M. Wythe, F. De Carlo, A.J. Davenport, A.R. Trueman, In situ X-ray tomography of intergranular corrosion of 2024 and 7050 aluminium alloys, Corros. Sci. $52 \quad$ (2010) 3855-3860. doi:10.1016/j.corsci.2010.08.026. 


\section{FIGURE CAPTIONS}

Figure. 1. Schematic representation of the FSW process.

Figure. 2. Cross section of the friction stir welded joint. The dashed red boxes indicate the regions were EBSD maps were acquired.

Figure. 3. EBSD analysis, grain boundary, orientation image, and Kernel average distribution maps of (a) base material, (b) stirred zone, (c) TMAZ at the advancing side, (d) TMAZ at the retreating side.

Figure. 4. (a) Boundary distribution and (b) variation of Kernel average misorientation of the different regions.

Figure. 5. SEM images showing the distribution and clustering of coarse intermetallic particles of the AA2024 alloy in the BM (a) and in the friction stir weldment: SZ (b), HAZ (c), TMAZ(AS) (d) and (f) and TMAZ(RS) (e) and (g).

Figure. 6. Macrographs showing the colour evolution of the regions of the friction stir weldment of the AA2024 during agar test. The image labeled "after" corresponds to the sample surface after $24 \mathrm{~h}$ of test, when the agar-agar gel was removed.

Figure. 7. OCP measurements for $1 \mathrm{~h}$ of immersion in $0.1 \mathrm{M} \mathrm{Na} \mathrm{SO}_{4}+1 \mathrm{mM} \mathrm{NaCl}$, for base metal (BM), heat affected zone (HAZ), thermomechanically affected zone (TMAZ), and stir zone (SZ), in advancing (A) and retreating (R) sides.

Figure. 8. OCP after $24 \mathrm{~h}$ of immersion in $0.1 \mathrm{M} \mathrm{Na} \mathrm{SO}_{4}+1 \mathrm{mM} \mathrm{NaCl}$, for the thermomechanically affected zone (TMAZ), in advancing (A) and retreating (R) sides. Figure. 9. Nyquist diagrams after $12 \mathrm{~h}$ of immersion in $\mathrm{Na}_{2} \mathrm{SO}_{4} 0.1 \mathrm{M}+\mathrm{NaCl} 1 \mathrm{mM}$, for: thermomechanical affected zone (TMAZ), in advancing (A) and retreating (R) sides. Figure. 10. SVET maps of the surface of the friction stir welded AA2024-T3 alloy showing the evolution of corrosion during immersion test in $5 \mathrm{mM} \mathrm{NaCl}$ solution. 
Figure. 11. (a) SVET map for $24 \mathrm{~h}$ and (b-d) corresponding SEM images of the regions marked in (a).

Figure. 12. LEIS maps of the surface of friction stir welded AA2024-T3 alloy showing the evolution of corrosion in $\mathrm{Na}_{2} \mathrm{SO}_{4} 0.1 \mathrm{M}+\mathrm{NaCl} 1 \mathrm{mM}$ solution. 2-D image corresponding to the diagram acquired after $24 \mathrm{~h}$, the dashed red lines indicate the presumed limit of the TMAZ. Acquisition frequency: $5 \mathrm{~Hz}$.

Figure. 13. SEM images of the (a) SZ-TMAZ-HAZ (AS) and (b) SZ-TMAZ-HAZ (RS) of the friction stir welded AA2024-T3 alloy after immersion in $\mathrm{NaCl}$ solution for 12 hours.

Figure. 14. SEM images (a-c) of the TMAZ(RS) of the friction stir welded AA2024-T3 alloy after immersion in $\mathrm{NaCl}$ solution for 2 hours. (d) EDS spectrum of the IMC particle indicated by an arrow in (b).

Figure. 15. SEM images (a-c) of the TMAZ(RS) of the friction stir welded AA2024-T3 alloy after immersion in $\mathrm{NaCl}$ solution for 12 hours showing different corrosion features. (d) EDS spectrum of the framed region depicted in (c). 
FIGURES

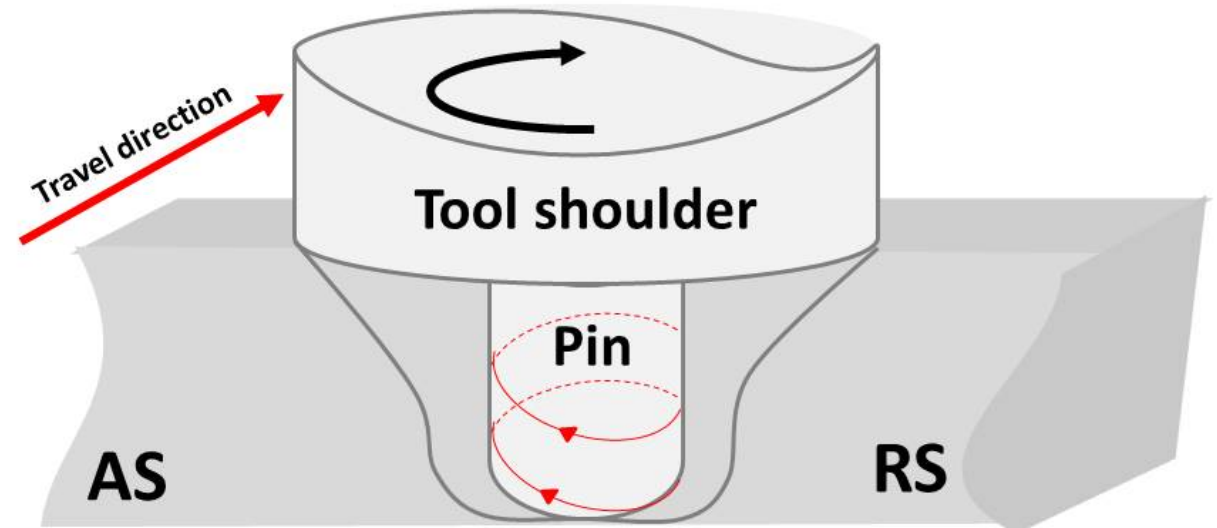

Figure. 1. 


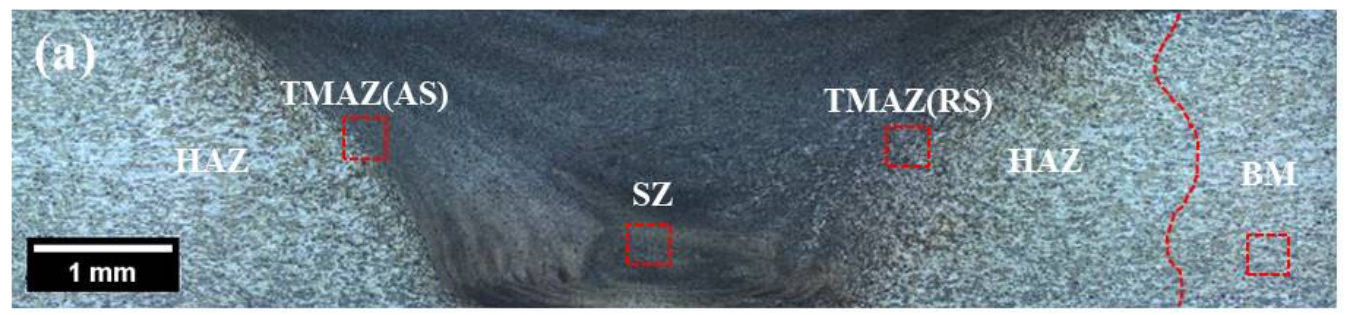

Figure. 2. 


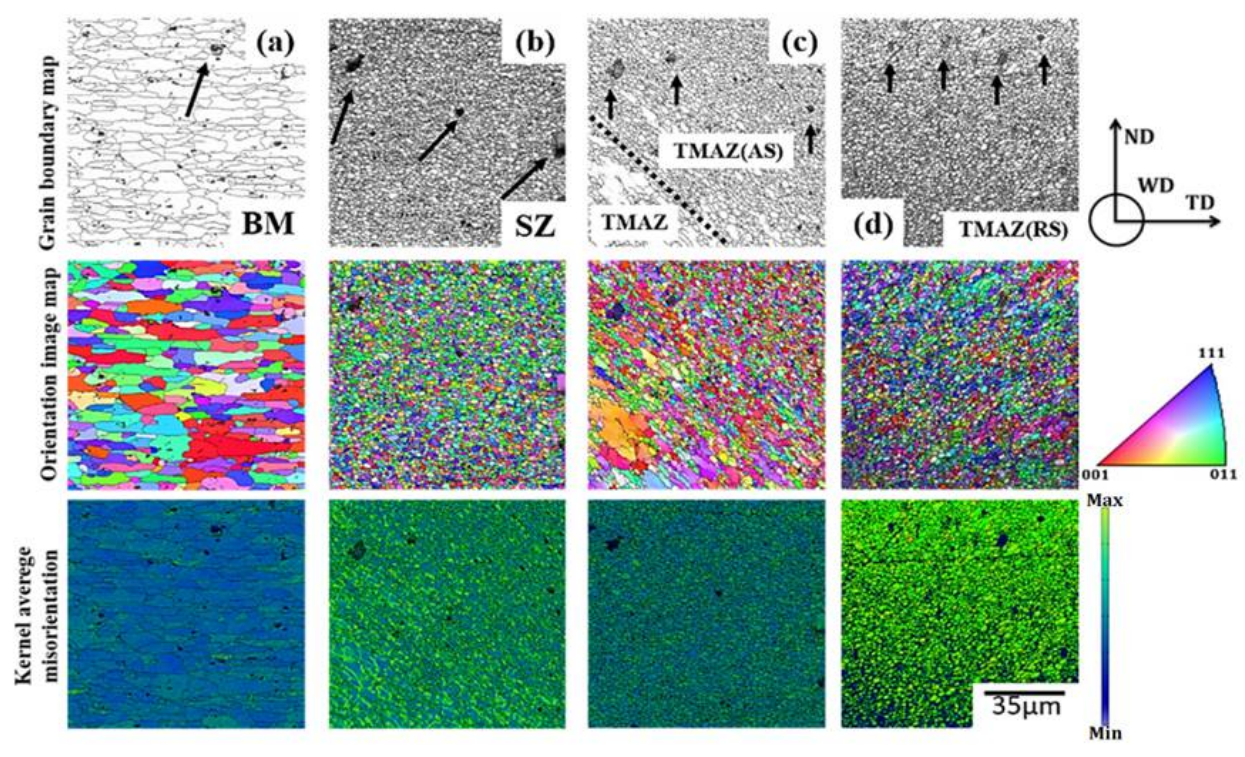

Figure. 3. 


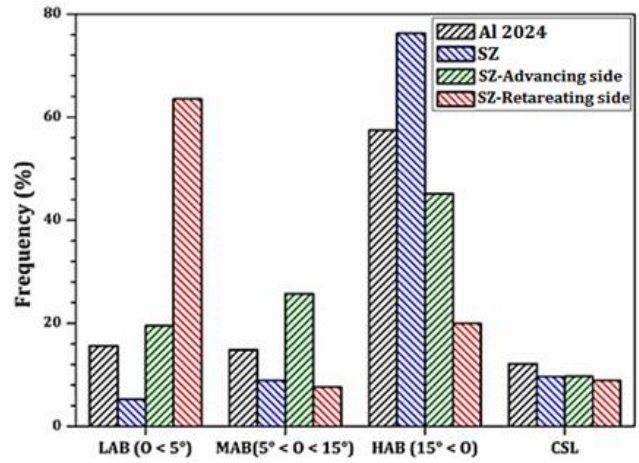

(a)

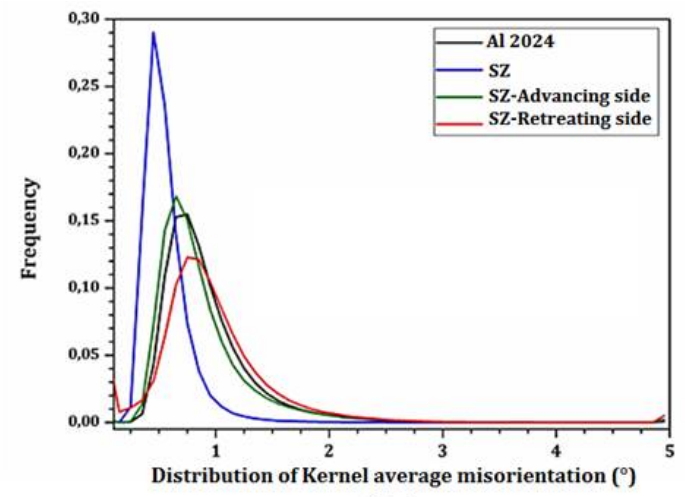

(b)

Figure. 4. 


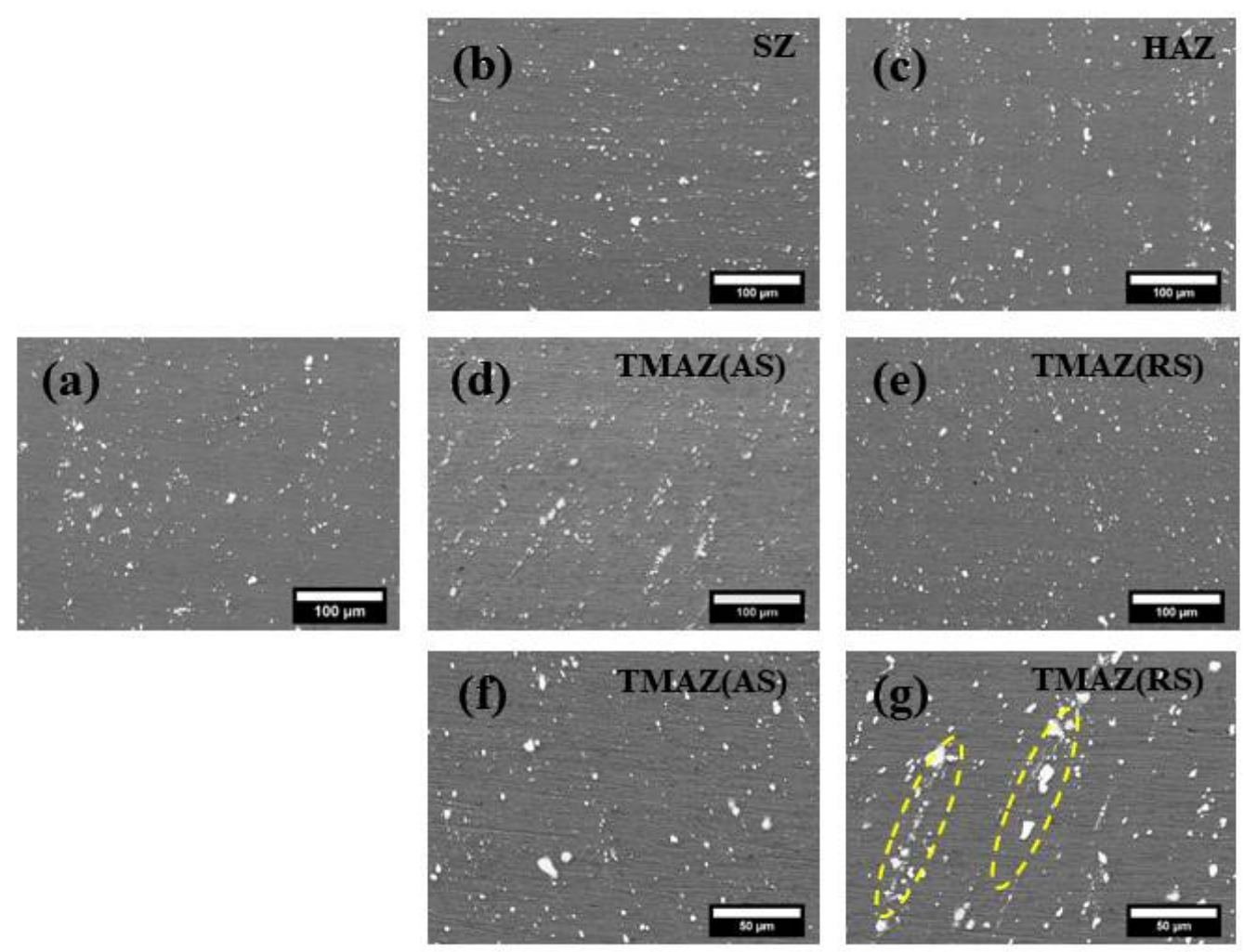

Figure. 5. 


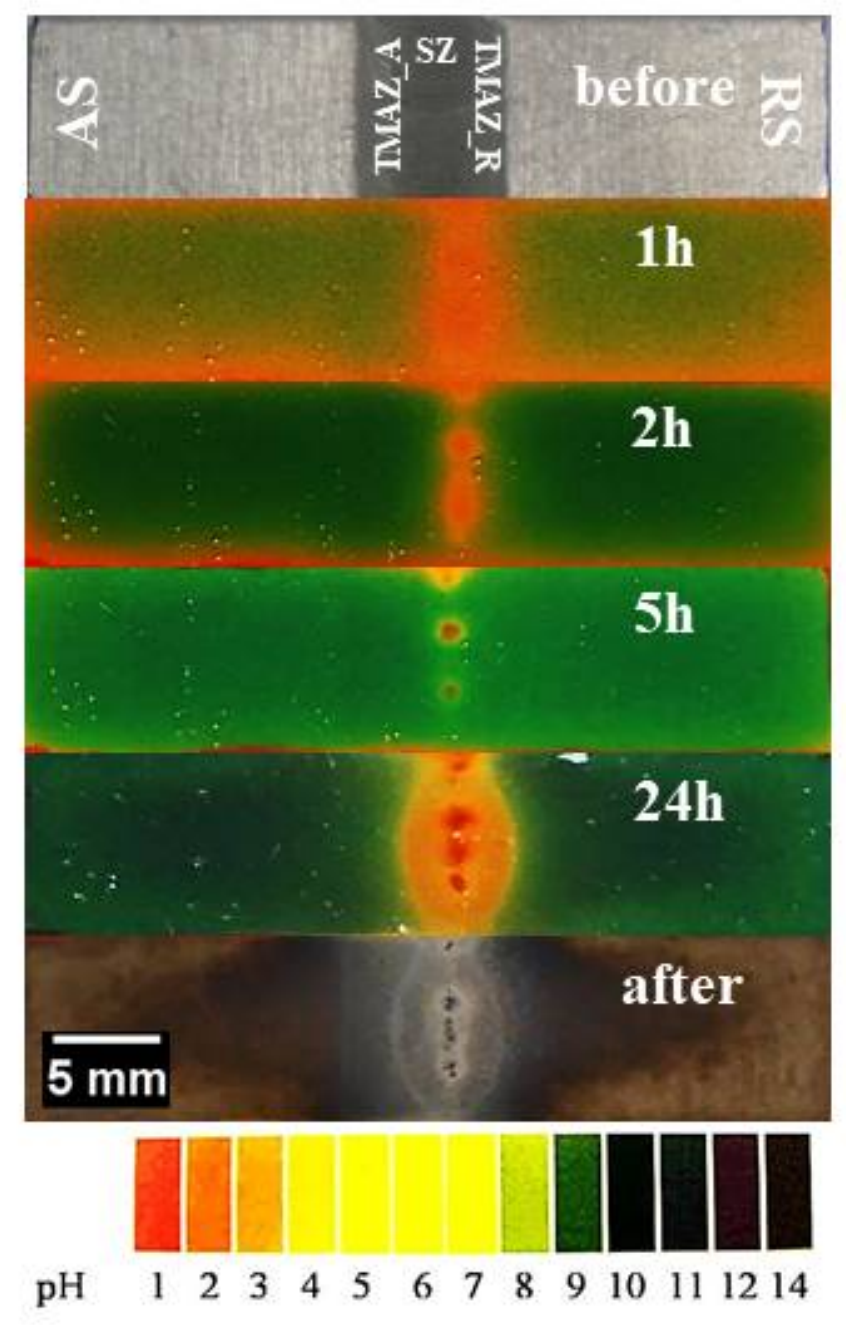

Figure. 6. 


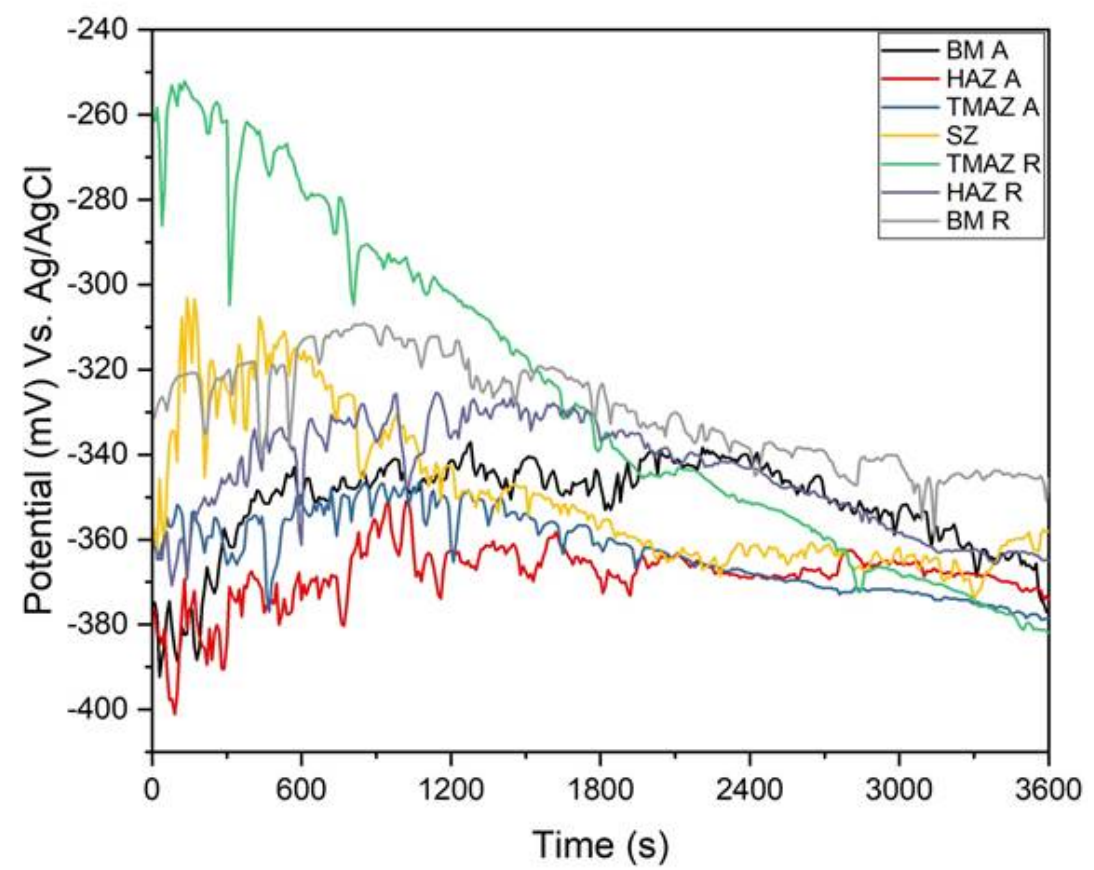

Figure. 7. 


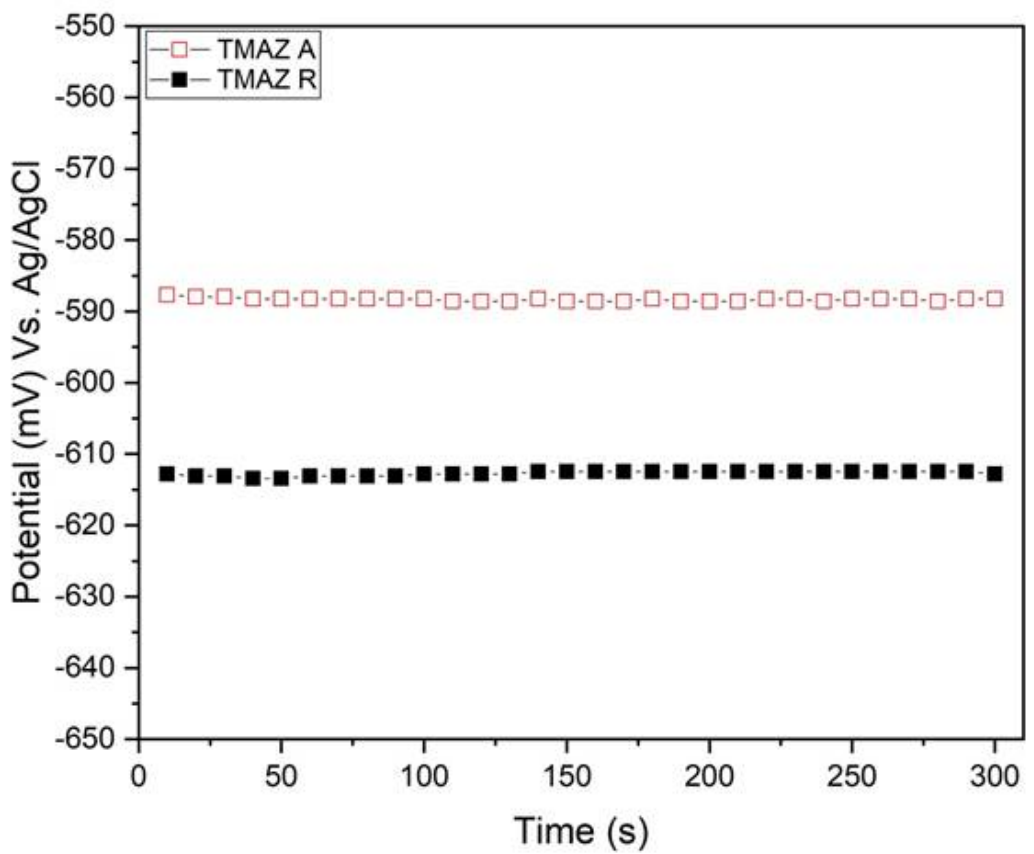

Figure. 8. 


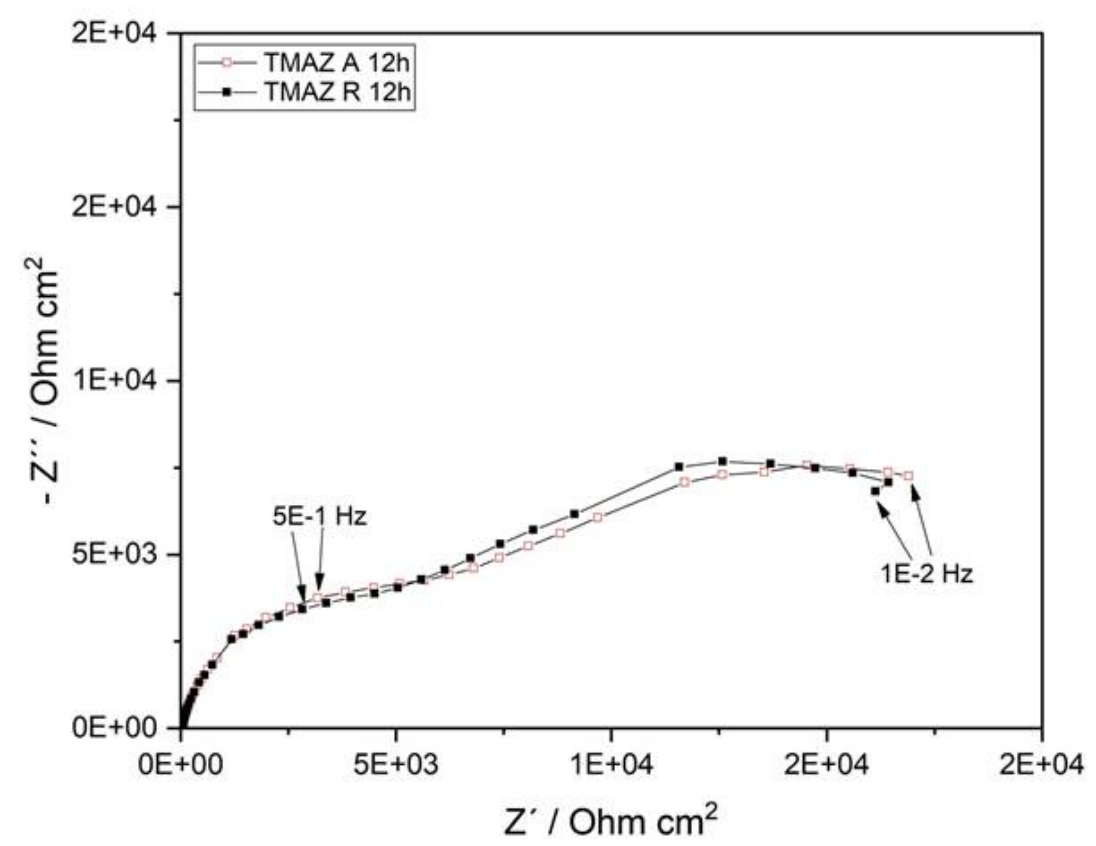

Figure. 9. 


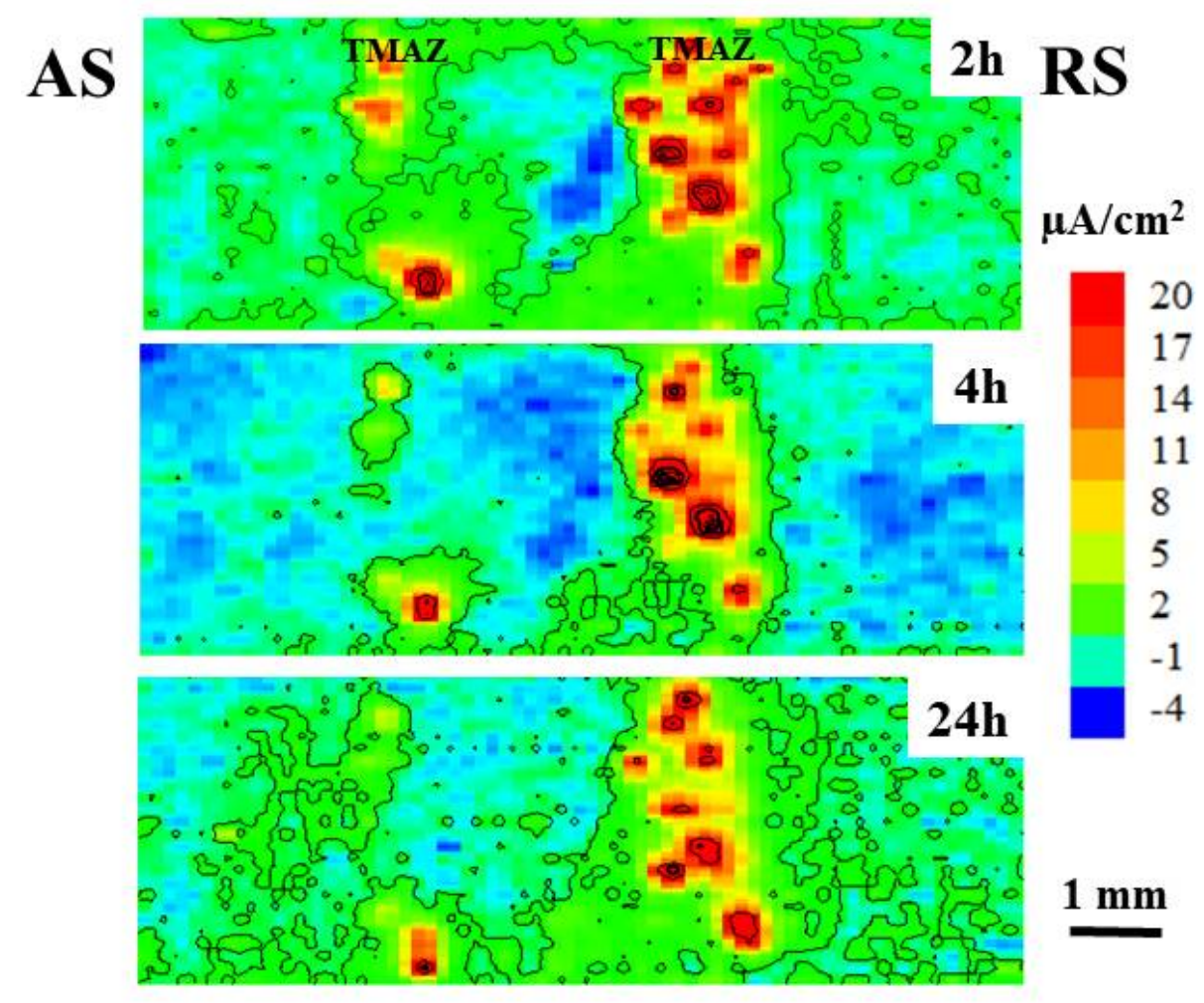

Figure. 10. 


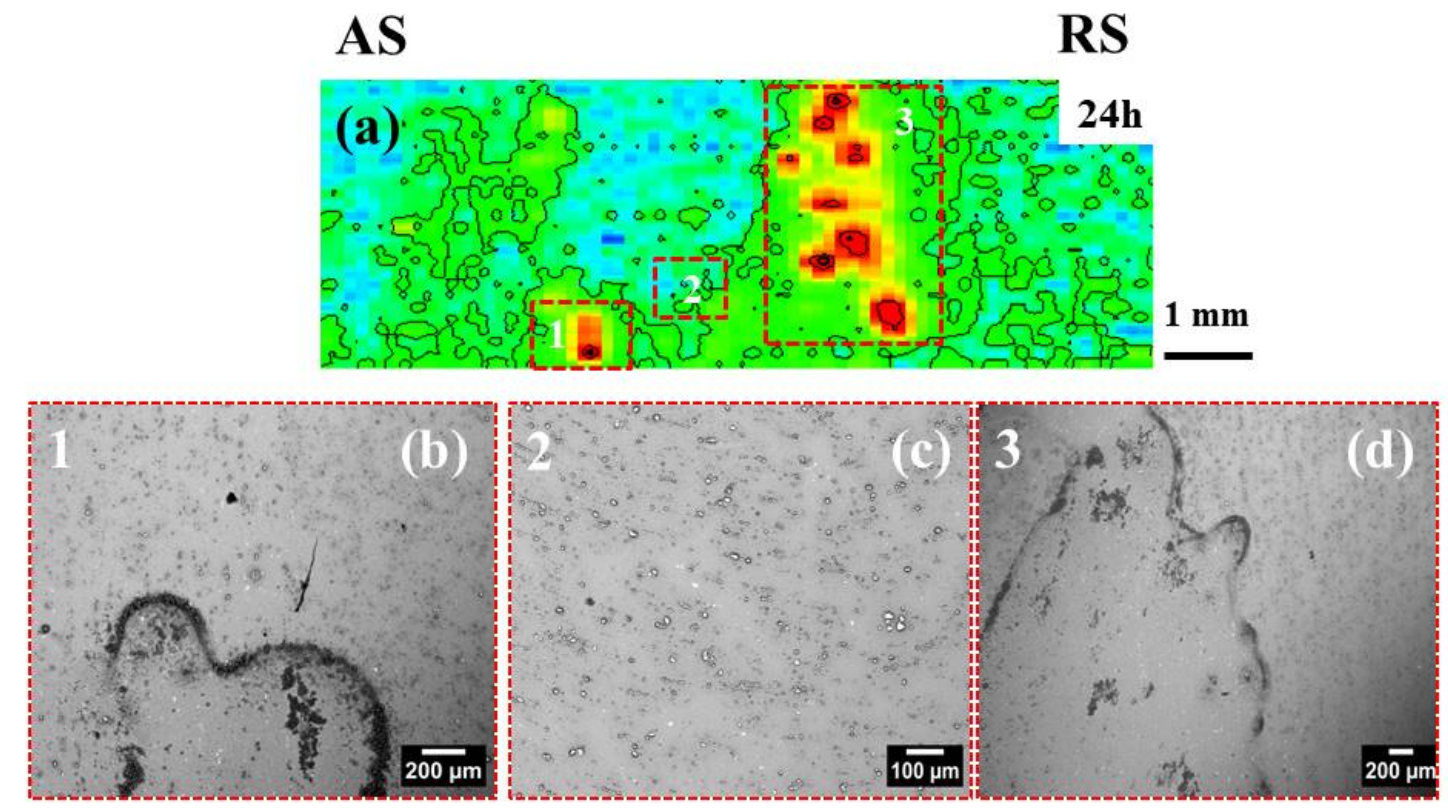

Figure. 11. 


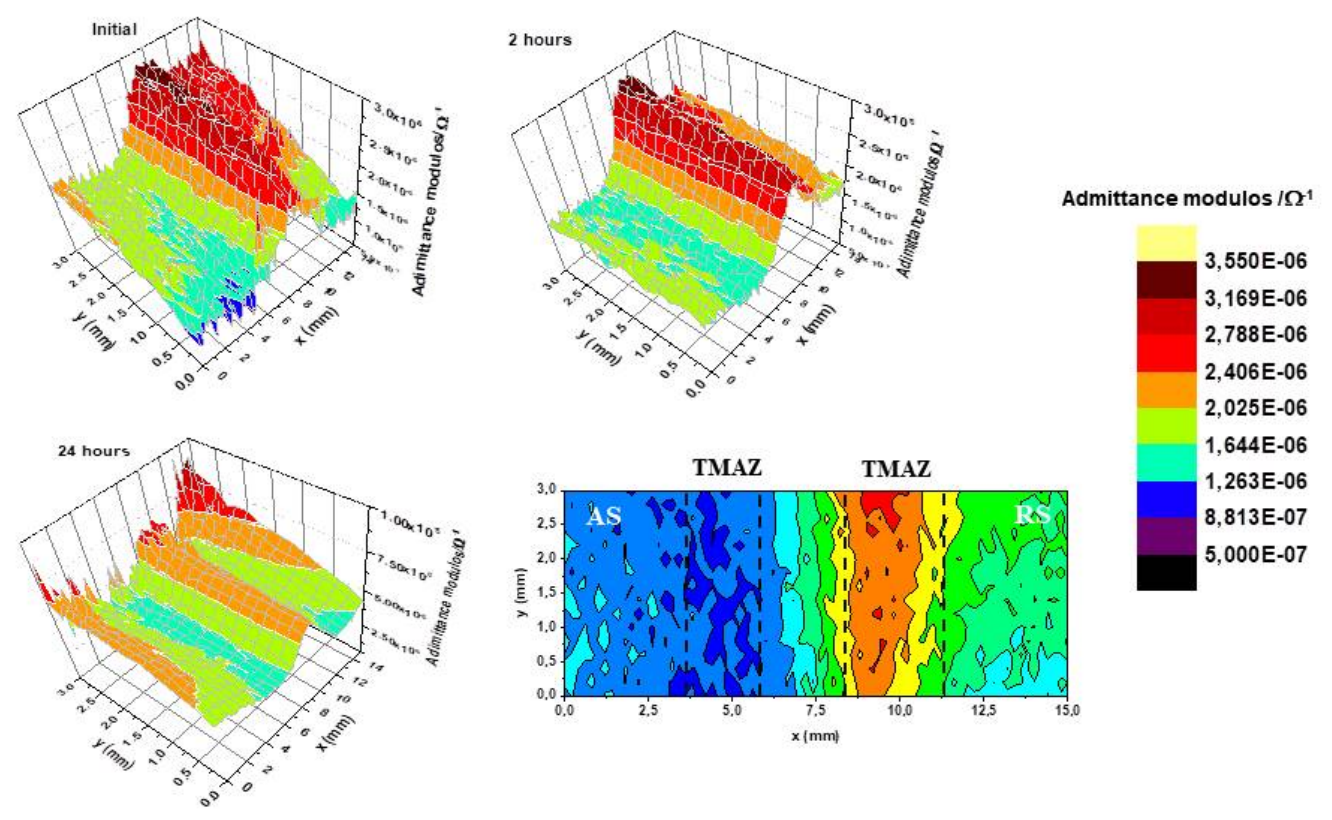

Figure. 12. 


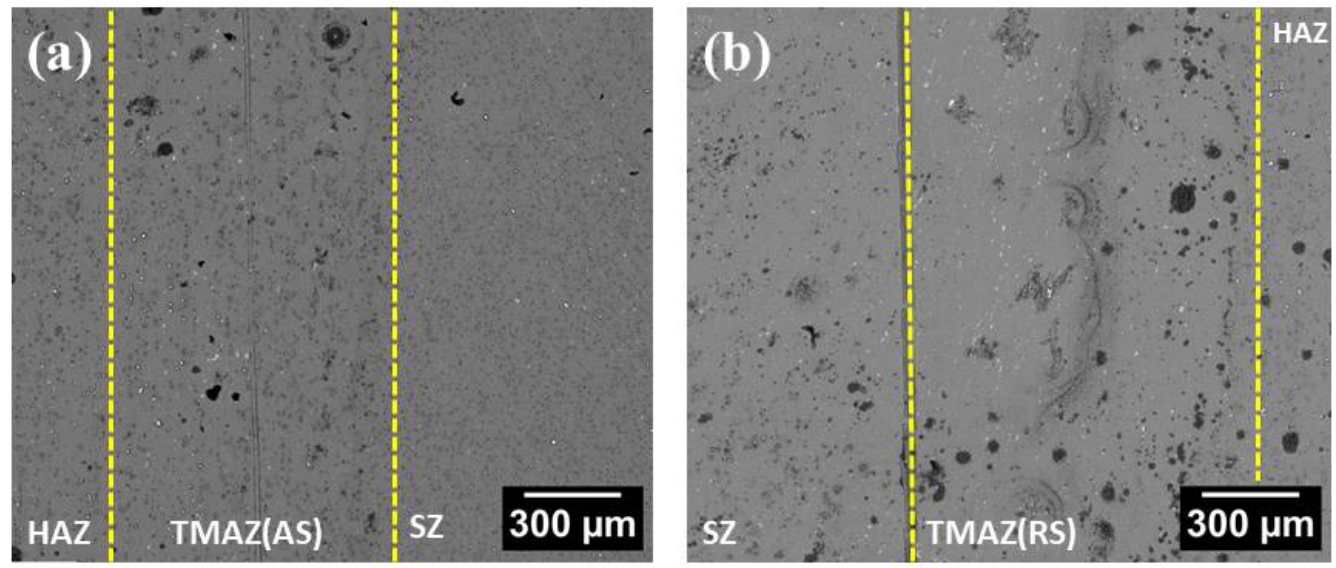

Figure. 13. 

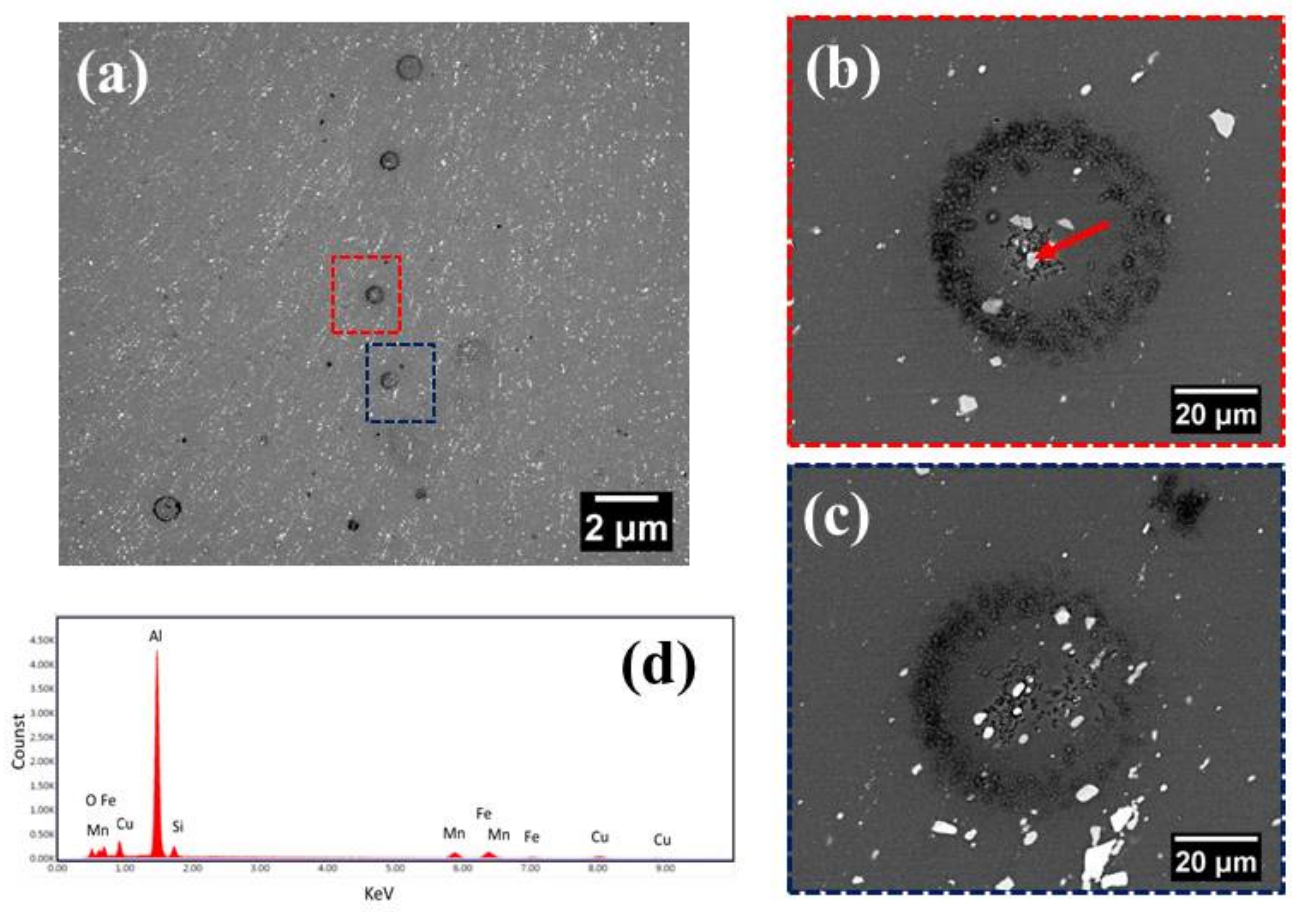

Figure. 14. 

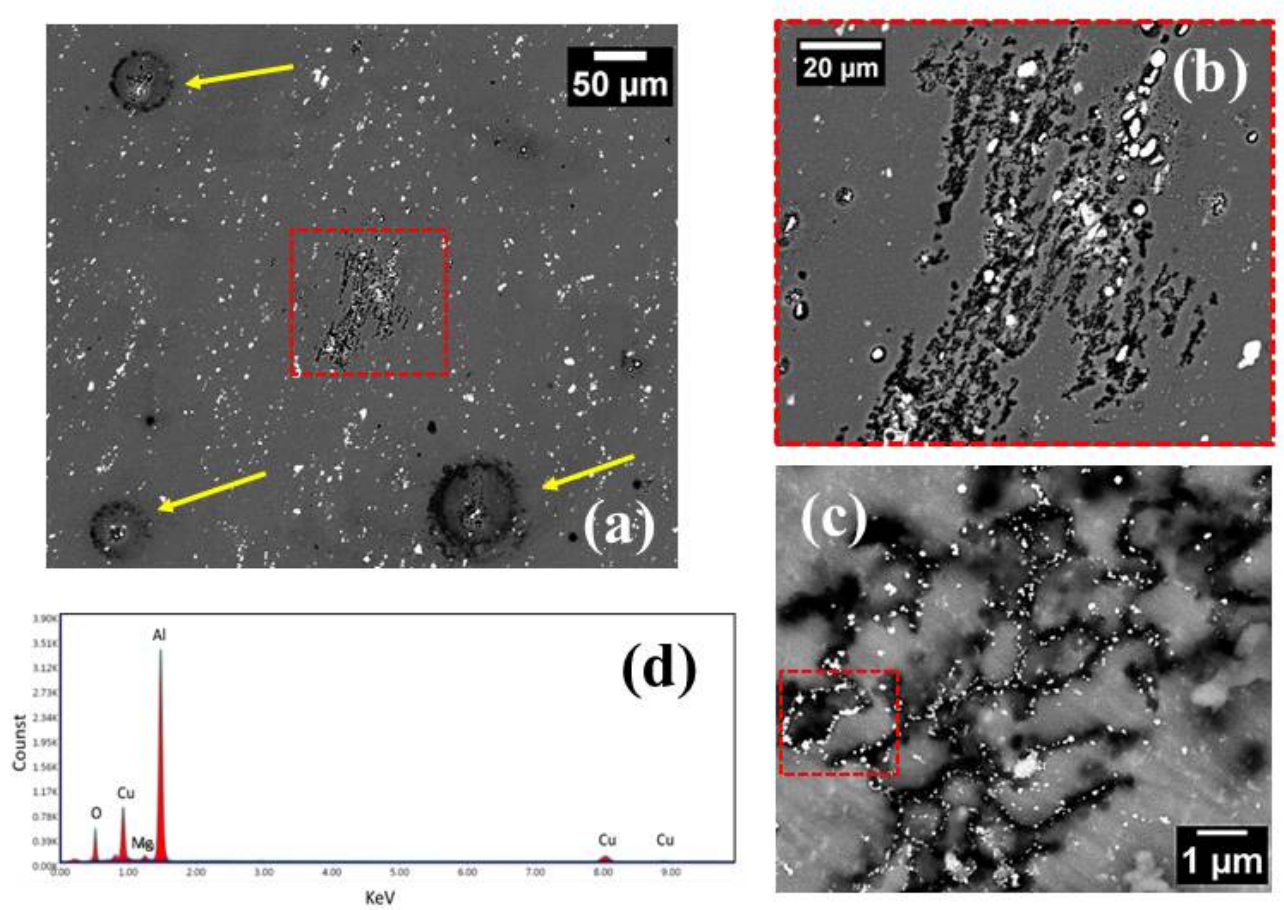

Figure. 15. 